\title{
Pushing it up the Agenda: Promoting the Importance of Physical Activity amongst Pregnant Women
}

\author{
Cotter $\mathrm{L}^{1}$, Smith $\mathbf{R}^{1 *}$, Hamish Reid ${ }^{1}$ and Mackillop $\mathrm{L}^{2}$ \\ ${ }^{1}$ Department of Sport and Exercise Medicine, Oxford University Hospitals NHS Trust, UK \\ ${ }^{2}$ Women's Centre, Oxford University Hospitals NHS Foundation Trust, UK
}

*Corresponding author: Ralph Smith, Department of Sport and Exercise Medicine, Nuffield Orthopaedic Centre, Oxford University Hospitals NHS Trust, Windmill Road, Oxford OX3 7LD

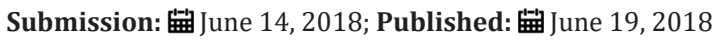

\section{Introduction}

Pregnancy provides an exciting opportunity to make positive lifestyle changes. As a major life event, pregnancy is considered an important 'teachable moment' and women receive routine advice regarding smoking cessation, adjusting dietary habits and abstinence from alcohol. For many, it may be considered a 'teachable moment' where women are advised to consider smoking cessation, adjusting dietary habits and abstinence from alcohol. Regular contact with healthcare professionals (HCP) at this time offers a unique opportunity to facilitate and consolidate desired behaviour change. The promotion of behavioural change such as smoking cessation is widespread in everyday practice amongst midwives. However, despite the overwhelming published evidence that regular moderate intensity physical activity (PA) during pregnancy has a positive impact, this lifestyle advice tends to receive less attention [1-3]. Currently, HCPs lack confidence and clarity to deliver this message. There is a need to educate and ensure that all HCPs caring for pregnant women have the knowledge and selfassurance to promote phsyical activity.

\section{Benefits of PA for pregnant women}

Benefits range from reducing the risk of gestational diabetes, hypertensive disorders and excessive gestational weight gain to improving cardiovascular fitness [1-4]. In addition, women will gain the benefits that all adults receive from keeping active (reduced risk of type 2 diabetes; cardiovascular disease; falls, depression and dementia; and cancer of colon and breast, together with improvements in sleep and mood) [5]. Furthermore, no evidence of adverse maternal or infant outcomes were identified from systematic reviews, suggesting that PA is safe in pregnancy [1-3]. Adapting these changes in behaviour during pregnancy may influence a woman's and her families' PA level across their life course. Effective interventions could provide an unique opportunity for population change [6].

\section{Common barriers to activity and lack of confidence amongst HCP}

Despite these known benefits and lack of adverse outcomes, the majority of pregnant women do not reach the recommended level of PA, and levels further decline through pregnancy [7]. Common barriers include lack of time and facilities, concerns about social disapproval, symptoms such as fatigue and nausea and worries about the safety of PA for the fetus [8-10]. HCPs have a key role to dissipate these barriers, facilitate and motivate women to increase or stay active during pregnancy [11]. Whilst advice from HCPs is welcomed by pregnant women [12] women often receive inconsistent, vague and conflicting guidance [9]. This is explained by many HCPs lacking the confidence, knowledge [13] and resources to deliver appropriate PA advice. They feel underresourced, uncertain regarding safety concerns and inadequately trained [14].

\section{Addressing this lack of consistency and confidence}

In 2017, a significant step forward was taken to address this lack of clarity in the UK. The Chief Medical Officers (CMOs) commissioned the development of an infographic designed for HCPs to use with pregnant women to facilitate PA brief advice. An expert committee was assembled. They were tasked to review all available evidence. Subsequently, evidence-based PA recommendations were created. These recommendations are succinctly summarised on an infographic and explained in more detail on an accompanying FAQ document, which can be found on the gov.uk website [3]. Whilst, the creation of the infographic is a useful tool and helps to clarify 
the recommendations, there is a need to disseminate and educate staff. The skills to deliver effective brief PA advice not only require adequate knowledge but awareness of barriers, empathy and the use of individual-centred behaviour change techniques $[15,16]$.

The lack of PA knowledge and education amongst maternity teams is neither completely surprising nor unique. Until recently general PA education was lacking on many undergraduate and postgraduate education curriculums [17]. Encouragingly, Webb et al have shown that a 60 minutes PA training intervention to nurses can increase the frequency of very brief advice given to cancer patients [18]. Furthermore, specific to maternity, compact training for midwives has been shown to improve self-reported knowledge and confidence levels in giving PA and dietary advice [19].

With this in mind, authors from this group, as part of the Moving Medicine project at the Oxford University Hospitals NHS Foundation Trust, developed a short education session which has been delivered to over 30 HCPs. The sessions focus on the infographic's recommendations actions and the use of communication techniques (based on motivational interviewing themes) to deliver brief advice to motivate women. Questionnaires prior to the start of the session and at two weeks follow up focused on participant's self-reported confidence to discuss PA with pregnant women. The educational sessions have resulted in improvements in participant's confidence levels and have led to an increased rate of PA discussion during consultations (from $31 \%$ to $76 \%$ self-reported rate). This is an early indication that these short education sessions are helpful in disseminating the message regarding the importance of PA for pregnant women and increasing the frequency of brief advice given.

\section{Conclusion}

HCPs must seize on the unique opportunity of pregnancy as a receptive and influential time in a woman's life to promote PA and facilitate behavioural change. HCPs must have the appropriate knowledge to deliver the advice of the CMOs PA and pregnant women's recommendations, be confident to use the infographic tool and the skills to deliver brief advice to this population. PA needs to be moved up the priority list of lifestyle advice given to pregnant women.

\section{Acknowledgement}

We would like to acknowledge the UK Chief Medical Officers' Physical Activity Expert Committee for Physical Activity and Pregnancy along with all staff members involved the Oxford University Hospital Foundation Trust physical activity feasibility project, part of the Moving Medicine project commissioned by Public Health England and the Faculty of Sport and Exercise Medicine (FSEM) and funded by Sport England with Money from the National Lottery.

\section{References}

1. Group IWMiPi-WC (2017) Effect of diet and physical activity based interventions in pregnancy on gestational weight gain and pregnancy outcomes: meta-analysis of individual participant data from randomised trials. BMJ 358: j3119.
2. Group UoOPAaPS (2016) Physical Activity and Pregnancy Infographic Study Interim Report.

3. Smith R, Reid H, Matthews A, Calderwood C, Knight M, et al. (2018) Infographic: physical activity for pregnant women. Br J Sports Med 52(8): 532-533.

4. (2015) ACOG Committee Opinion No. 650: Physical activity and exercise during pregnancy and the postpartum period. Obstet Gynecol 126(6): e135-142.

5. Health Do (2011) Start Active, Stay Active. In: Health Do (Ed).

6. Haas S (2008) Trajectories of functional health: the 'long arm' of childhood health and socioeconomic factors. Soc Sci Med 66(4): 849861.

7. Borodulin KM, Evenson KR, Wen F, Herring AH, Benson AM (2008) Physical activity patterns during pregnancy. Med Sci Sports Exerc 40(11): 1901-1908.

8. Van Mulken MR, McAllister M, Lowe JB (2016) The stigmatisation of pregnancy: societal influences on pregnant women's physical activity Behaviour. Cult Health Sex 18(8): 921-935.

9. Weir Z, Bush J, Robson SC, McParlin C, Rankin J, et al. (2010) Physical activity in pregnancy: a qualitative study of the beliefs of overweight and obese pregnant women. BMC Pregnancy and Childbirth 10: 18.

10. Harrison AL, Taylor NF, Shields N, Frawley HC (2018) Attitudes, barriers and enablers to physical activity in pregnant women: a systematic review. J Physiother 64(1): 24-32

11. Warren L, Rance J, Hunter B (2017) Eat Well Keep Active: Qualitative findings from a feasibility and acceptability study of a brief midwife led intervention to facilitate healthful dietary and physical activity behaviours in pregnant women. Midwifery 49: 117-123.

12. Willcox JC, Vander Pligt P, Ball K, Wilkinson SA, Lappas M, et al (2015) Views of women and health professionals on mhealth lifestyle interventions in pregnancy: a qualitative investigation. JMIR Mhealth Uhealth 3(4): e99.

13. Hopkinson Y, Hill DM, Fellows L, Fryer S (2018) Midwives understanding of physical activity guidelines during pregnancy. Midwifery 59: 23-26.

14. McParlin C, Bell R, Robson SC, Muirhead CR, Araújo Soares V (2017) What helps or hinders midwives to implement physical activity guidelines for obese pregnant women? a questionnaire survey using the theoretical domains framework. Midwifery 49: 110-116.

15. Lindqvist M, Persson M, Mogren I (2018) Longing for individual recognition-Pregnant women's experiences of midwives counselling on physical activity during pregnancy. Sex Reprod Healthc 15: 46-53.

16. Brown MJ, Liddle D, Hill AJ, Stockdale JME (2013) Motivating pregnant women to eat healthily and engage in physical activity for weight management: an exploration of routine midwife instruction. Evidence Based Midwifery 11(4): 120-127.

17. Dunlop M, Murray AD (2013) Major limitations in knowledge of physical activity guidelines among UK medical students revealed: implications for the undergraduate medical curriculum. Br J Sports Med 47(11): 718720 .

18. Webb J, Foster J, Poulter E (2016) Increasing the frequency of physical activity very brief advice for cancer patients. Development of an intervention using the behaviour change wheel. Public Health 133: 4556.

19. Basu A, Kennedy L, Tocque K, Jones S (2014) Eating for 1, Healthy and Active for 2; feasibility of delivering novel, compact training for midwives to build knowledge and confidence in giving nutrition, physical activity and weight management advice during pregnancy. BMC Pregnancy Childbirth 14: 218 
Creative Commons Attribution 4.0 International License

For possible submissions Click Here

\section{EPMR}

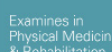

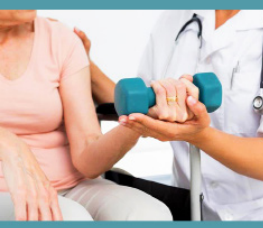

Examines in Physical Medicine and Rehabilitation: Open Access

\section{Benefits of Publishing with us}

- High-level peer review and editorial services

- Freely accessible online immediately upon publication

- Authors retain the copyright to their work

- Licensing it under a Creative Commons license

- Visibility through different online platforms 